\title{
Molecular mechanisms of intensive care unit-acquired weakness
}

\author{
S. Bloch*,\#, M.I. Polkey", M. Griffiths" and P. Kemp*
}

ABSTRACT: Intensive care unit-acquired weakness (ICUAW) is an increasingly recognised and important clinical consequence of critical illness. It is associated with significant morbidity and mortality. The aetiology of this disease is not well understood. The purpose of this article is to review our understanding of the molecular pathogenesis of ICUAW in the context of current knowledge of clinical risk factors and aetiology.

Key features of the disease are loss of muscle mass resulting from a shift in the dynamic balance of muscle protein synthesis and breakdown and a reduction in force-generating capacity. These alternations are secondary to neuropathy, disruption of the myofilament structure and function, a disrupted sarcoplasmic reticulum, electrical inexcitability and bioenergenetic failure.

As knowledge and understanding of ICUAW grows, potential therapeutic targets will be identified, hopefully leading to multiple strategies for prevention and treatment of this important condition.

\section{KEYWORDS: Critical illness, intensive care unit-acquired weakness, muscle wasting}

ntensive care unit-acquired weakness (ICUAW) is an increasingly recognised and important clinical consequence of critical illness. It is associated with significant morbidity and mortality. Recent clinical studies have defined the clinical risk factors for this condition, described its epidemiology and led the drive for a better understanding of its pathology.

ICUAW is characterised by skeletal muscle wasting and weakness. In severe cases, complete paralysis can occur and up to $30 \%$ of patients are left with long-term disability [1]. ICUAW is associated with failure to wean from mechanical ventilation, increased length of stay in the intensive care unit (ICU) and hospital, and in some studies with increased risk of death [2-4]. At least $25 \%$ of patients who are intubated for more than 7 days develop ICUAW [2] as do up to $100 \%$ of those who have severe sepsis and systemic inflammatory response syndrome (SIRS) [5]. It is difficult to know the prevalence of ICUAW, as definitions and diagnostic techniques vary widely. However, data from the worldwide Surviving Sepsis Campaign suggest that 750,000 patients a year develop severe sepsis in North America, with similar numbers estimated for Europe [6]; 170,000 patients per year are admitted to critical care in the UK [7] and an estimated $6 \%$ of all critical care beds are occupied by weaning patients [8]; consequently, when combined with an estimated incidence of $25 \%$ in these patients, ICUAW is a huge clinical and financial burden, for which, as yet, there is no effective treatment or prevention strategy.

Sepsis, SIRS and multi-organ failure are major clinical risk factors for ICUAW [9, 10]. Uncontrolled hyperglycaemia is also a significant risk factor. Other clinical factors supported by less conclusive evidence include: glucocorticoids (GCS) and neuromuscular blocking agents [10]. ICUAW develops in patients who are usually immobile as a result of their critical illness and often impaired consciousness. While there is a well-recognised syndrome of disuse atrophy, the evidence that immobility is a critical factor in ICUAW is indirect and models comparing skeletal atrophy in disuse and sepsis show distinct differences [11, 12].

\section{CHANGES IN MUSCLE PHENOTYPE AND FUNCTION IN PATIENTS WITH ICUAW}

The muscles of patients with ICUAW demonstrate a range of alterations. Muscle strength depends on the force-generating capacity of the muscle and the muscle mass, and both are thought to be affected in ICUAW. Clinically, this

\section{AFFILIATIONS}

*Dept of Molecular Medicine, National Heart and Lung Institute, Imperial College, London, and

\# Royal Brompton Hospital, NIHR Respiratory Biomedical Research Unit, London, UK.

CORRESPONDENCE

S. Bloch

Department of Molecular Medicine National Heart and Lung Institute Imperial College London

London

SW7 2AZ

UK

E-mail: susannah.bloch@

doctors.org.uk

Received:

May 272011

Accepted after revision:

Aug 312011

First published online:

Sept 292011 
manifests as muscle wasting preceded by abnormal muscle electrophysiology. A key feature of the disease is a marked loss of thick myosin filaments with disruption to the actin:myosin ratio [13-16] and a disruption of the myofilament organisation of the tissue. Normally, skeletal muscle exists in a regular striated pattern, formed by the organisation of the myofilaments, which is required for the generation of force in an organised manner. In the muscle of patients with ICUAW, there is a significant disruption to this organisation, which is likely to contribute to the reduction in the force-generating potential of the muscle $[17,18]$. As seen in muscle wasting associated with ageing and other atrophic states, such as chronic inflammation or starvation, a shift towards fast fibres expressing type 2 myosin has been reported in ICUAW $[14,19]$. In addition, these type 2 fibres demonstrate greater atrophic changes and are thought to waste more rapidly $[14,20]$, suggesting increased sensitivity of type 2 fibres to the multifactorial insult of critical illness. However, robust and reproducible data on the differential vulnerability of muscle fibres to ICUAW and histological analysis of the changes associated with this disease are lacking. A recently developed pig model examining different potential risk factors for ICUAW failed to show a difference in major histocompatibility complex expression profiles or disruption to the actin:myosin ratio [12], but as suggested by the authors, this may be due to the relatively short duration of the study. A more prolonged model and studies in chronic ICUAW patients are needed to describe the cellular and histological changes that may occur in ICUAW.

Other mechanisms that contribute to the loss of power include neuropathy, a reduction in energy generation by the muscle through insulin resistance and mitochondrial dysfunction, dysregulation of calcium handling and electrical inexcitability. These are discussed individually later in this article.

Much of the evidence for the molecular pathways involved in the pathogenesis of ICUAW is inferred from in vitro and animal models of muscle wasting. Animal models that are commonly used to simulate immobility are denervation, hind limb suspension or cast immobilisation; and models of sepsis induced by endotoxin, pro-inflammatory cytokines or caecal ligation and puncture (CLP). These models are often used in combination with administration of high-dose dexamethasone or neuromuscular blocking agents. Some of these studies should be interpreted with caution, since supra-physiological doses are sometimes used. The insult of ICUAW is multifactorial, but as discussed above the evidence from clinical studies for risk factors other than for sepsis/inflammation is contradictory and variable. Therefore, while these models are limited, their use has helped to identify candidate pathways involved in ICUAW, which now need to be validated in clinical studies. However, clinical studies are notoriously difficult to carry out successfully, in part due to the heterogeneity of the critical illness. Hence, there is currently a lack of human ICUAW studies examining the molecular pathology.

The purpose of this article is to review the current understanding of the molecular pathogenesis of ICUAW in the context of what is known about clinical risk factors and aetiology. As the knowledge and understanding of this condition grows, potential therapeutic targets will be identified, hopefully leading to multiple strategies for prevention and treatment of this important condition.

\section{MUSCLE MASS IN ICUAW}

Muscle mass is determined by the balance between muscle protein synthesis and breakdown. This allows for rapid responses to changing demands. In hypertrophic responses, both protein synthesis and degradation pathways can be increased, with a greater increase in the rate of protein synthesis; similarly, the reverse can be true during atrophic responses. The disruption in the balance of synthesis and catabolism was first demonstrated in 1968 in response to denervation (a model that is commonly used to represent ICUAW) [21]. In healthy muscle, this flexibility allows for repair of muscle fibres and release of amino acids for gluconeogenesis. In normal healthy adults, the rate of synthesis matches the rate of protein degradation such that there is turnover, but no net change in muscle protein. In critical illness, this balance is upset, with the overall catabolic state resulting from a shift towards dominance of the atrophic pathways (fig. 1).

\section{Upregulation of muscle protein breakdown in ICUAW}

Protein degradation occurs through a number of pathways, including the ubiquitin-proteasome (UP) pathway, caspase and calpain activity, and autophagy. Of these, the UP pathway is thought to be the major mechanism of proteolysis [22].

This process is highly complex and specific, which enables the regulated degradation of specific proteins. In essence, the pathway involves tagging the protein(s) to be degraded with ubiquitin moieties. This tag targets the protein to the proteasome, a large multi-protein complex that degrades proteins to their constituent amino acids. The addition of ubiquitin to a protein involves three enzymes. Ubiquitinactivating protein (E1) and ubiquitin carrier protein (E2) prepare the ubiqutin molecule for conjugation. The ubiquitin ligases (E3) then catalyse the ligation of ubiquitin to specific proteins [22]. In addition to having target specificity, these ubiquitin ligases can also be restricted in their expression in different tissues: for example, atrogin and muscle ring finger-1 (MuRF1) are muscle specific [22-24]. Of these two proteins, MuRF1 targets thick myosin filaments and, therefore, in view of the specific thick filament loss, it is likely to be especially important in ICUAW [25].

The UP pathway is activated in a number of muscle atrophic conditions, including the response to inactivity and inflammation [22]. Both MuRF1 and atrogin were first identified in relation to disuse atrophy $[23,24]$ and animal knockout models for these ligases are resistant to atrophy caused by denervation [24]. Similarly, unloading muscle in space flight increased activation of the UP pathway in rats [26]. Expression of UP proteins is regulated by the transcription factor nuclear factor $(\mathrm{NF})-\kappa \mathrm{B}$, which is in turn negatively regulated by the inhibitor of NF- $\kappa \mathrm{B}(\mathrm{I} \kappa \mathrm{B})$. A possible mechanism for the role of sepsis and inflammation in the upregulation of the UP pathway is the increased ubiquitination and degradation of $\mathrm{I} \kappa \mathrm{B}$ seen in response to the pro-inflammatory cytokine tumour necrosis factor (TNF) $-\alpha[27,28]$. NF- $\kappa B$ activation also increases the expression of MuRF1, suggesting a role in muscle-specific 


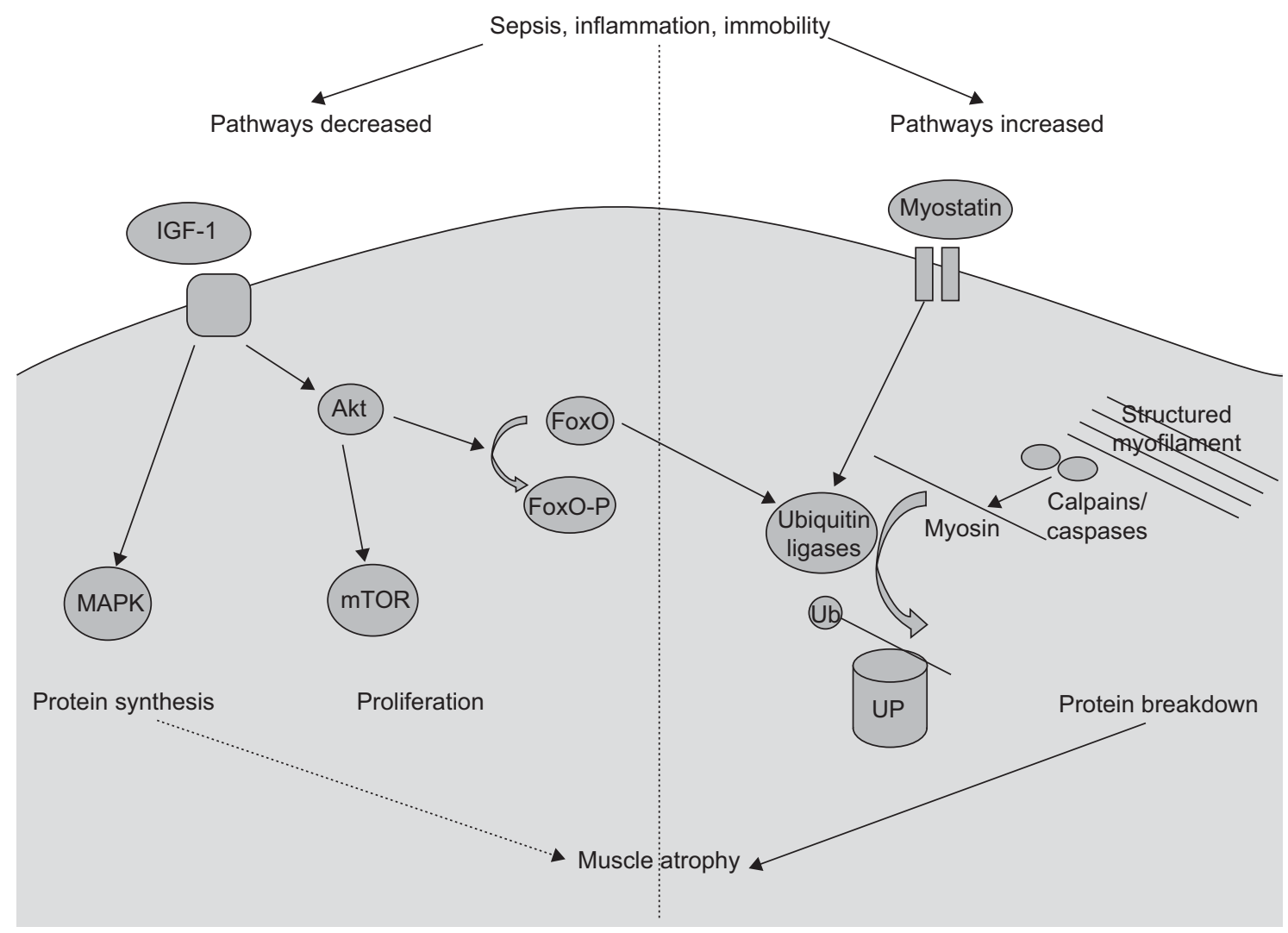

FIGURE 1. Schematic of the main molecular pathways balancing muscle protein synthesis and breakdown. In intensive care unit-acquired weakness, sepsis, inflammation and immobility result in muscle atrophy by shifting this balance in favour of muscle protein breakdown. IGF-1: insulin-like growth factor 1; MAPK: mitogen-activated protein kinase; mTOR: mammalian target of rapamycin; FoxO: forkhead box protein; FoxO-P: phosphorylated FoxO (inactive); UbP: ubiquitin proteasome; Ub: ubiquitin.

atrophy [29]. Although activation of NF- $\mathrm{KB}$ is often associated with inflammation, a 10-fold increase in the activity of an NF$\kappa \mathrm{B}$ reporter was demonstrated after 7 days of muscular unloading in rat soleus muscle [30]. Sepsis induced by CLP increases mRNA of both muscle-specific ligases [31] and this increase can be inhibited by GCS receptor antagonists, suggesting a potential mechanism for GCS potentiating muscle wasting in sepsis. These data are consistent with the known role of GCS in myopathy and with the increase in the UP pathway that GCS can elicit [32]. Furthermore, denervation has been shown to increase sensitivity to GCS-induced disruption of the balance between protein synthesis and breakdown [21]. However, the importance of this response in ICUAW is not clear, as the clinical evidence for GCS as a factor in ICUAW is inconclusive [10].

Increased protein ubiquitination in the muscle of ICUAW patients has been demonstrated alongside damage to myosin filament structure [33] and increased UP mRNA has also been shown in septic patients' muscle [34]. More recently, increased activity of the proteasome and direct evidence for increased muscle proteolysis have been shown in both respiratory $(30 \%$ increase compared with controls) and limb (45-55\% increase compared with controls) muscle of septic patients in the ICU [35].

Other atrophic factors also increase the expression of MuRF1 and atrogin. For example, myostatin, a member of the transforming growth factor (TGF)- $\beta$ family and a potent negative regulator of muscle mass [36], increases many atrophic pathways including MuRF1 and atrogin [37]. Excessive myostatin activity is implicated in hereditary muscular dystrophy and has been linked in males to muscle wasting in cardiac failure, cancer and HIV [38-41]. Myostatin signalling is also elevated by GCS [42] and is negatively correlated with daily activity [43], however, as yet myostatin has not been shown to be relevant in ICUAW. Lysosomal activity has also been implicated in sepsis-induced muscle wasting and treatment of septic rats with leupeptin (an inhibitor of the lysosomal protease cathepsin B) has been shown to inhibit the associated muscle wasting. In this study, activation of cathepsin B was dependent on the action of prostaglandins as indomethacin inhibited both atrophy and the activation of cathepsin B [44]. Other intracellular proteases may also be involved in ICUAW, but whether they contribute directly to increase protein turnover rather than disruption of the myosin-actin complex (discussed later in this article) is not clear.

\section{Downregulation of muscle protein synthesis in ICUAW}

In parallel with increased muscle breakdown is a relative decrease in muscle protein synthesis and repair. A number of studies in both humans and animals have shown a marked decrease in muscle protein synthesis immediately after surgery, which persists for at least 3 days [45]. The duration of this reduction is not clear as some studies have shown that the effect lasts for up to 30 days [45], while others have 
demonstrated an increase in muscle protein synthesis in ICUAW-like conditions after 1 week. However, even in these studies, the rate of protein breakdown still exceeded that of protein synthesis, resulting in catabolism [46, 47]. Decreased protein synthesis also occurred in response to both sepsis and immobility [48-50] and, in healthy volunteers, in response to endotoxin infusion [51].

The major signalling pathway thought to drive protein synthesis in muscle is the insulin-like growth factor (IGF)-1/ Akt signalling cascade $[52,53]$. In this system, IGF-1 binds to its receptor, a serine/threonine kinase that can activate the mitogen-activated protein kinase (MAPK) cascade, increasing myoblast proliferation, or the Akt pathway, which activates protein synthesis by activating mammalian target of rapamycin (mTOR), and inhibiting glycogen synthase kinase- $3 \beta$, itself an inhibitor of protein synthesis [52,54]. In addition to stimulating protein synthesis, Akt also inhibits the expression of MuRF-1 and atrogin by phosphorylating forkhead box (Fox)O proteins $[55,56]$ and thereby inhibits atrophy.

It has long been accepted that mediators of sepsis decrease IGF-1 levels, in both in vitro and in vivo models [48]. For example, IGF-1 is reduced in the diaphragm of septic mice [57]. Consistent with this, CLP-induced muscle protein loss in mice can be prevented by implanting a small IGF-1 pellet into the muscle [58]. Such changes in IGF-1 secondary to sepsis directly correlate with decreased muscle protein synthesis, via reduced mTOR activation and expression of translational proteins [59]. Similarly, Akt signalling and FoxO phosphorylation were decreased and MuRF-1 and atrogin expression increased in a septic rodent model [60].

IGF-1 is also induced by muscle contraction and reduced with disuse [61] suggesting a second mechanism by which IGF-1 may be suppressed in patients with ICUAW. Together, these data make it likely that IGF-1 pathways will be downregulated in ICUAW but direct evidence is currently lacking and one recent study failed to identify differences between serum IGF-1 levels in patients who developed ICUAW from and levels in those who did not [62]. This observation demonstrates the problems in translating laboratory-based experiments into clinical studies. Also demonstrating the limitation of experimental models is the observation that some human studies of immobilisation have shown a greater decrease in protein synthesis compared with the increase in breakdown [63], which is the converse of that observed in many rodent models [64].

Another potential mechanism suggested as the cause of reduced protein synthesis is anabolic resistance. It is postulated that in the setting of critical illness, muscle exhibits a syndrome of anabolic resistance whereby it is unable to use the supply of amino acids available to respond to anabolic signals or maintain adequate protein synthesis [64]. This may be due to damage to the microcirculation secondary to sepsis and an ensuing reduction in supply of oxygen, nutrients and growth hormones to the muscle [65].

\section{Reduction in force-generating capacity in ICUAW}

Loss of muscle mass obviously contributes to long-term weakness in ICUAW patients. However, many ICUAW patients become weak prior to detectable muscle wasting and an abnormal electromyogram (EMG) is predictive of ICUAW and poor outcomes [66]. This early weakness also occurs in animal models of sepsis, where a reduced force-generating capacity has been measured in the diaphragm independent of any muscle wasting [67, 68]. Similar responses have been found in peripheral muscles [12], for example a greater than $50 \%$ reduction in force generation of both limb and respiratory muscles in response to endotoxin, again prior to any evidence of muscle wasting, has been reported [69]. As these changes occur within hours of the onset of sepsis and prior to any signs of fibre wasting, the mechanism is likely to be independent of the UP pathway and reduced protein synthetic pathways described above [70]. Again, many factors are likely to play a part, but five major mechanisms have been proposed: 1) neuropathy; 2) altered myocyte structure and myofilament integrity; 3) sarcoplasmic reticulum dysfunction; 4) electrical inexcitability; and 5) bioenergetic failure and oxidative stress (fig. 2).

\section{Neuropathy and nerve activity}

Patients with ICUAW often have abnormal nerve conduction (more typically axonal loss than prolonged conduction time [71]) and the disease was originally postulated as "critical illness neuropathy" [72]. The effect of denervation of muscle is complex because nervous stimulation is required for the maintenance of muscle phenotype as well for contraction. It is important to note when considering the direct effect of inflammatory mediators on the muscle that denervation can increase the tissue's sensitivity to these factors. This was initially demonstrated in animal models. Sciatic nerve severing combined with high-dose steroids leads to myosin loss and fibre inexcitability in rats, which was reversed by re-innervation [73]. However, given the rapidity of the reduction in force-generating capacity that occurs in ICUAW, it seems unlikely that it is due solely to neuropathy.

Traffic in the nerve is especially pertinent to ventilator-induced diaphragm dysfunction (VIDD) since the aim of ventilatory support is to reduce the work of the respiratory muscles. It is difficult to separate the aetiology of VIDD and ICUAW. Systemic sepsis and inflammation clearly affect the diaphragm as much as other muscles and some of the evidence that we have discussed in respect to ICUAW has been generated from rodent diaphragm models. However, not only is the diaphragm a different muscle with a different phenotype, but the muscle is

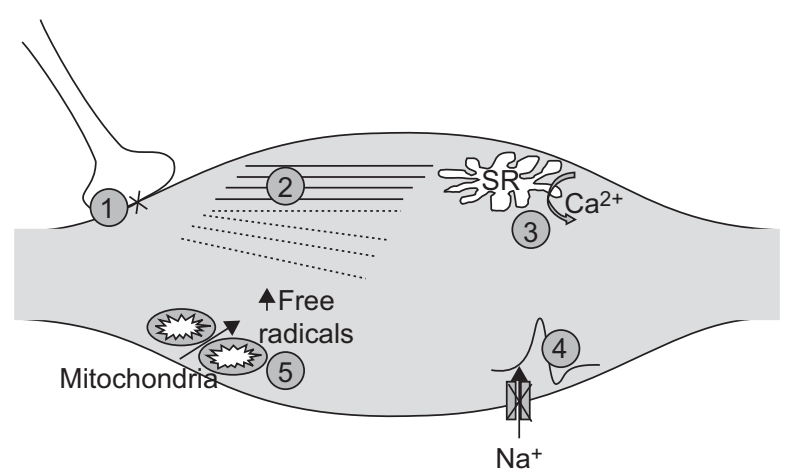

FIGURE 2. Representation of potential mechanisms reducing the forcegenerating potential of skeletal muscle in intensive care unit-acquired weakness. 1: neuropathy; 2: altered myocyte structure and myofilament integrity; 3 : sarcoplasmic reticulum (SR) dysfunction; 4: electrical inexcitability; 5: bioenergetic failure. 
never immobile. Instead, it is either active during respiratory function, or in the case of total mechanical ventilation, it is subjected to varying degrees of passive stretch. Therefore, VIDD is not purely an ICUAW of the diaphragm. A full discussion of VIDD is beyond the scope of this article and the condition has been reviewed recently elsewhere (e.g. in [74]); at this point, it is sufficient to note only that diaphragm weakness is common among intubated patients [75, 76] and may increase with duration of ventilation [77], and that modes of ventilation that require continued [78] (or at least in rodents, even intermittent [79]) phrenic activity may prevent or attenuate VIDD.

\section{Myocyte structure and myofilament integrity: maximal force generation requires an organised myofilament structure and function}

Disruption of myofilament structure, for example induced by TNF- $\alpha$ [80], or of the mechanisms regulating the contractile process are therefore potential contributors to reduced force generation. As myosin and actin are the major components of the contractile filaments, proteins that cleave them are potential disruptors of myofilament structure. Such proteins include the calpains and caspases, some of which are thought to be required to cleave myosin, thereby enabling its removal from the sarcomere prior to ubiquitination and proteolysis. These proteins therefore act not only to increase the pool of proteins ready to be broken down for amino acids, but also to disrupt the structure of the myofibrils, which reduces the contractile potential of the fibre [81, 82].

Calpains are calcium-dependent cysteine proteases that under basal conditions are inactive and whose role in health and disease is not fully understood. There are at least 14 different calpain proteins, some of which are ubiquitously expressed (e.g. $\mu$ - and m-calpain), whereas others are tissue-specific: for example calpain 3, also known as p94, is muscle specific [81]. In muscle atrophy, $\mu$ - and m-calpain are upregulated [81], whereas p94 is linked to certain muscular dystrophies [83] and may be downregulated in denervation atrophy [84]. Increased calpain-dependent protein degradation in sepsis is well established [85]. Calpains are thought to be activated predominantly by intracellular calcium, which can be increased in sepsis (see later in this review and [86]). Activation of calpains also reduces Akt phosphorylation and may therefore contribute to decreased protein synthesis [87]. Increased calpain activity decreased contractile function in septic models of muscle wasting [88, 89]; thus it is possible that activation of calpains contributes to the early loss in force generation, as well as contributing to proteolysis.

The role of caspases in cell death and apoptosis is well recognised, but they have recently been implicated in muscle atrophy and are now recognised as contributing to sepsisinduced myopathy. Caspases can be activated via an intrinsic mitochondrial pathway or via extrinsic receptors that can also activate apoptosis [89], with caspase 3 the final common mediator. Caspases also disrupt myofilament structure, suggesting a role in the loss of force generation. Accordingly, in mice the loss of force-generating capacity of the diaphragm induced by endotoxin was accompanied by an increase in caspases and was attenuated by caspase inhibitors [70].

Although the role for both caspases and calpains in ICUAW appears convincing, most of these studies have been carried out on diaphragm muscle from animal models and there are as yet no human data to confirm this.

\section{Altered function of the sarcoplasmic reticulum}

Appropriate levels and changes in intracellular calcium in muscle are critical for contraction both because of the direct effects of calcium on myosin ATPase activity in the contraction cycle and through the regulation of glycolytic and oxidative energy metabolism. However, calcium is also an important regulator of protein breakdown, activating calpains and ubiquitination. In skeletal muscle, the sarcoplasmic reticulum contributes to calcium homeostasis and is the organelle responsible in muscle for calcium binding and release. Sepsis has marked effects on calcium homeostasis, increasing intracellular calcium in limb skeletal muscle [86], reducing calcium binding in isolated sarcoplasmic reticular membrane [90], decreasing release of calcium from the sarcoplasmic reticulum and increasing the sensitivity of contractile proteins to calcium [91]. Several of these activities (e.g. increased intracellular calcium and increased myofibrillar sensitivity to calcium) may be predicted to increase force generation. However, the net effect of all of the changes appears to be a reduction in force generation, implying that the effects of proteolytic activation and reduced calcium release from the sarcoplasmic reticulum are the dominant effects. In addition to these animal studies, the serum from critically ill patients who developed ICUAW reduced calcium-activated force generation in permeabilised muscle fibres by blocking calcium release. The serum fraction used was low molecular weight, $<30 \mathrm{kDa}$, suggesting a role for inflammatory cytokines in this process [92].

\section{Electrical inexcitability}

It has been postulated that sepsis and critical illness result in a general systemic syndrome of electrical inexcitability [17]. Both muscle and nerve are relatively inexcitable in septic patients [93-95] and this observation has led to the idea that critical illness myopathy and neuropathy coexist as a result of a common pathology. Although an axonal neuropathy exists in patients with ICUAW, the nerve histology is often normal, suggesting functional rather than structural abnormalities [17, 96]. As a number of cytokines are known to be neurotoxic, the increase in inflammatory cytokines found in ICUAW patients may contribute to the electrical excitability, although the scant data supporting this contention are difficult to interpret. For example, exposure of cultured neuronal cells to serum from patients with ICUAW resulted in neurotoxicity in 12 out of 16 cases in one study, but $50 \%$ of the control sera also caused neurotoxicity [97]. Membrane alterations also occur in ICUAW, resulting in chronic depolarisation and reduced excitability of the nerve [93], and therefore functional denervation of the adjacent muscle [98]. ICUAW is therefore likely to be a result of both neuronal and muscular damage. Cardiac inexcitability in sepsis has also been demonstrated by reduced ECG potentials in septic patients [99] lending weight to the argument for a generalised syndrome of inexcitability.

The mechanisms for this inexcitability have been extensively investigated. However, the majority of these studies again have been in animal and in vitro models. One common model used is steroid- and denervation-induced myopathy, but as already discussed in this article, the evidence for these as aetiological 
factors is unconvincing. The resting membrane potential of muscle fibres is reduced in rat muscles that have been denervated. When denervation is then combined with exposure to GCS, the fibre is unable to produce action potentials [100]. The membrane itself is disrupted during sepsis (demonstrated by increased uptake of a low-molecular-weight dye that is not normally able to penetrate the membrane) and this alters the membrane potential [101]. In addition, inflammatory cytokines and GCS appear to cause acute hyperpolarisation of sodium channels, but in rat models of ICUAW, an overall reduction in sodium channel availability and sodium current are seen. The sodium channel is made up of two subunits, which in normally innervated muscle are both of the Nav1.4 isoform. In denervated muscle, an embryonic form (Nav1.5) is expressed and this leads to abnormal polarisation [102]. The sodium channels are voltage dependent, but in ICUAW, the normal function is shifted to a more negative threshold, which in animal models results in $99 \%$ of the available sodium channels being inactivated [102]. Therefore, the muscle fibre is unable to produce an action potential. Not all fibres are affected to the same extent and the percentage affected determines the severity of weakness [17]. Increased NaV1.5 has also been shown in a model of chronic sepsis in rats [103] and lipopolysaccharide interaction with and disruption of the channel has been demonstrated in vitro [104]. However, these effects have yet to be shown in ICUAW muscle.

Bioenergenetic failure, oxidative stress and glycaemic control

The combination of tissue hypoxia, insulin resistance and increased oxidative stress resulting from sepsis and critical illness leads to reduced glucose uptake and ATP formation, mitochondrial dysfunction and increased free radical production. This condition is termed bioenergenetic failure [105]. Glucose is a primary source of energy used by muscle. In the absence of sufficient glucose, muscle proteolysis occurs to provide amino acids for gluconeogenesis. The uptake of glucose into muscle is controlled by insulin-dependent stimulation of GLUT4 receptors. Insulin resistance in muscle associated with critical illness and sepsis reduces glucose uptake and ATP production and this results in mitochondrial dysfunction.

Hypoxia is also known to increase oxidative stress, and in healthy individuals and COPD patients, hypoxia has been shown to contribute to muscle wasting [106-108]. Many critically ill patients are also hypercapnic; indeed, this tendency will increase as physicians try to reduce barotraumas. Hypercapnia resulting in systemic and cellular acidosis results in further disrupted metabolic function, decreased ATP and increased free-radical production. These effects of hypercapnia reduce contractile force in both diaphragm and limb skeletal muscle [109-111]; thus, both hypoxia and hypercapnia may worsen bioenergenetic failure. However, direct evidence for the relative importance of gas exchange abnormalities in ICUAW is lacking.

A vicious cycle can occur within the mitochondria, in which sepsis-induced increased mitochondrial production of free radicals exacerbates mitochondrial dysfunction, leading to further radical production [112, 113] (fig. 3). Furthermore, animal models of endotoxin-induced sepsis suggest a direct suppressive effect on expression of genes encoding electron transport components, levels of mitochondrial proteins and

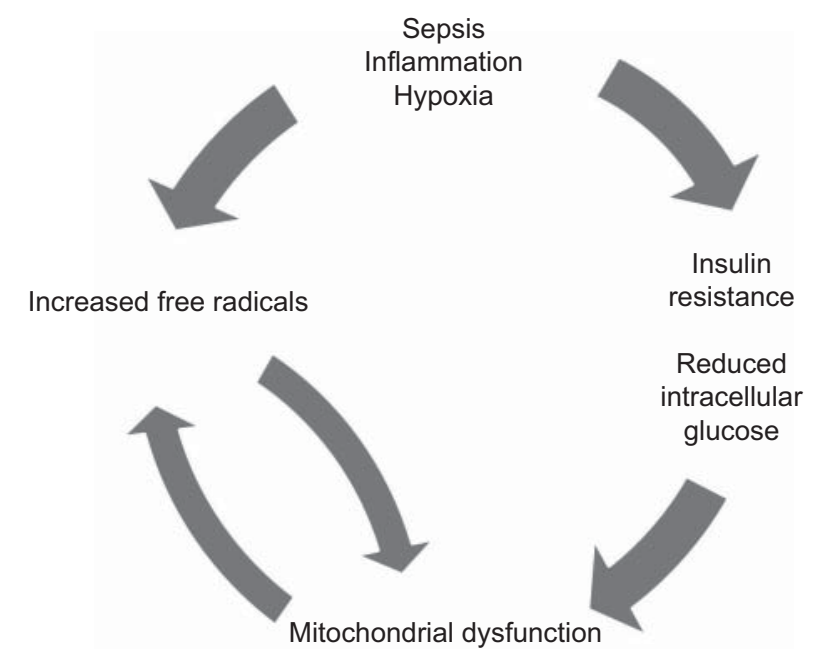

FIGURE 3. Mitochondrial dysfunction in bioenergenetic failure results in increased free radical production and, in turn, further mitochondrial damage.

mitochondrial oxygen consumption [112, 114, 115]. In a longerterm model of sepsis and organ failure, which may be more representative of the aetiology of ICUAW, a reduction in respiratory chain complex enzyme 1 was persistent [116]. A similar reduction in total mitochondrial activity was found in patients with multi-organ failure who had recently been diagnosed with severe sepsis but were now cardiovascularly stable [117]. This reduction was assayed as a reduction in total citrate synthase and respiratory complex enzymes I and IV in the muscle. However, there was no difference in the activity of complex I when compared to citrate synthase activity, suggesting that there was no deficiency in the electron transport capacity of individual mitochondria. Indeed, the relative expression of complex IV normalised to citrate synthase was significantly elevated. The remaining mitochondria demonstrated sustained mitochondrial protein synthesis and increased activity of superoxide dismutase; however, this was insufficient to maintain normal mitochondrial function [117]. Furthermore, microarray studies of these patients identified upregulation of transcription control genes responsible for regulation of mitochondrial biogenesis (nuclear respiratory factor (NRF) $2 \alpha /$ GA binding protein $(\mathrm{GABP})$ ) and network analysis studies have demonstrated oxidative stressrelated pathway activation [117]. Mitochondrial dysfunction in limb muscle in the acute setting of critically ill patients with septic shock and ICUAW has been observed and noted to be an independent risk factor for mortality [118]. However, not all studies in humans have shown mitochondrial dysfunction in muscle from critically ill patients [119].

In skeletal muscle, oxidative stress resulting from increased free radicals is implicated in many of the pathways potentially contributing to ICUAW, including those leading to total muscle protein loss [120,121] and reduced force-generating potential [112]. For example, oxidative stress increases ubiquitination of cellular proteins and also activity of the UP itself $[122,123]$. Similarly, both calpains and caspases can be induced by free radicals [88, 124, 125] and free radicals have been shown to cause sarcolemmal damage, which can be reduced by 
inhibition of nitric oxide synthase [101]. Finally, it has also been suggested that free radicals may interact directly with the contractile apparatus of the myofilament and by modifying the protein structure, reduce contractile potential [126].

In addition to mitochondria, the main sources of free radicals in muscle are sarcolemmal NAPDH oxidase and nitric oxide synthase [82, 112]. Free radical production is increased from each of these sources in sepsis [127-129]. In animal models, reversal of some of the pathological effects of sepsis can be achieved with free radical scavengers and inhibitors of free radical production $[88,130,131]$. Although the potential of antioxidant therapy has been demonstrated in some causes of muscle atrophy, as yet there is no evidence for this in ICUAW [132].

Tight glycaemic control with intensive insulin therapy in two large studies performed in surgical and medical ICUs reduced ICUAW from $49 \%$ to $25 \%$ [133] and from $50 \%$ to $38.9 \%$, respectively [134]. This therapy is currently the only pharmacological intervention that has been shown to be effective in prevention of ICUAW. The mechanisms for this are unclear, but it is thought that treatment with insulin helps to combat insulin resistance and, thereby, protects against bioenergetic failure [105]. Insulin therapy upregulates GLUT4 and hexokinase, resulting in increased insulin-controlled uptake of glucose into skeletal muscle and, therefore, more availability for mitochondrial function. In addition to providing tight glycaemic control, insulin has other potentially beneficial effects for skeletal muscle. Studies performed in animals or in vitro have shown that insulin is anabolic (by promoting protein synthesis [135] and inhibiting the UP [136]), is potentially anti-inflammatory [137], improves dyslipidaemia [138] and is neuroprotective [139]. Consistent with an anabolic role for insulin in humans, a post mortem study of critically ill patients has shown an increase in skeletal muscle protein concentration in those who were treated with intensive insulin therapy compared with controls [119]. Similar results have been shown in burns patients [135] and cancer cachexia [140]. Also, in a recent human study of patients with ICUAW where all participants were treated with intensive insulin therapy, IGF-1 and skeletal muscle protein synthesis were increased and protein breakdown pathways decreased compared with controls. [141]. However, in the above post mortem study, insulin was shown to protect the mitochondria of liver but not skeletal muscle [119], further demonstrating the difficulties of using in vitro and animal studies as models for ICUAW.

Another potentially beneficial effect of insulin is to counteract the hyperglycaemic effect of GCS and permit their antiinflammatory properties. Although the evidence for GCS as a risk factor for ICUAW is inconclusive, GCS-induced myopathy is well recognised and some studies have shown a large increase in relative risk of ICUAW with GCS exposure [142]. However, in the studies of HERMANS et al. [134] and VAN DEN BERGHE et al. [133], GCS were in fact found to be protective against ICUAW. Perhaps clinical studies that have not in the past controlled for insulin therapy, hyperglycaemia or insulin resistance are less relevant to current practice.

\section{IMMOBILITY}

Immobility is a difficult factor to quantify and control in critical illness, but it is likely to be a major contributor to the pathology of ICUAW. Critically ill patients are generally immobile for a number of reasons. These include cardiovascular instability, inability to mobilise and reduced level of consciousness. Disuse muscle atrophy outside the context of critical illness is well recognised and can be demonstrated in healthy volunteers [143]. In response to disuse, dramatic muscle atrophy and loss of muscle strength (of up to $5 \%$ ) are seen within the first week.

While unloading and immobilisation have been used in models of ICUAW, pure disuse muscle atrophy differs significantly from ICUAW. Cast immobilisation was shown in one study not to reduce force-generating capacity of the abductor pollicus muscle, but to reduce overall strength and cause muscle atrophy. By contrast, septic patients in the same study demonstrated marked reduction in force-generating capacity [11]. The reasons for this difference are unclear. However, together, critical illness and immobility result in even worse muscle damage. In a rat model where septic and control rats had one leg immobilised, comparison of immobilised and control legs revealed that the combination of the two treatments resulted in much more deranged neuromuscular function and atrophy than either sepsis or immobility alone [144]. One potential explanation may be that disuse drives a switch from slow, fatigue-resistant type 1 to type 2 fibres [19] rendering the muscle more sensitive to inflammatory insult; and as we have seen, it is the type 2 fibres that are most effected in ICUAW $[14,20]$.

Calcium homeostasis and free radical excess are also implicated in immobility-related muscle damage. Unloading increases free radical production and thus oxidative stress (as discussed earlier in this article) [145] and intracellular calcium concentrations (potentially via free radical damage to the sarcoplasmic reticulum) [146]. As muscle atrophies, each multinucleate fibre begins apoptosis by slowly reducing its number of nuclei [147]. Caspase 3 is central to this pathway and in rat hind-limb suspension models of wasting, increased caspase 3 activity was associated with a reduction in the number of nuclei per muscle fibre [148]. Immobility has also been shown to increase microvascular permeability, induce local insulin resistance and decrease antioxidant potential [149].

Neuromuscular blocking drugs obviously enforce immobility, a potential mechanism for their pathological effects in ICUAW. They too are often used in animal models to simulate the ICU condition [25]. But evidence has been inconclusive in clinical analysis and as with GCS, the use of these drugs is often unavoidable. Another potential mechanism is that prolonged exposure to high concentrations of neuromuscular blocking agents at the neuromuscular junction upregulates acetylcholine receptor expression. These receptors may be functionally immature and less sensitive to acetylcholine signalling [150].

Counteracting immobility is potentially a simple way to prevent ICUAW. Early rehabilitation with physiotherapy is likely to be beneficial $[151,152]$. Consequently, stimulation of muscle contraction with neuromuscular electrical stimulation (NMES) is increasingly being investigated as a method to reduce ICUAW. Several small studies have demonstrated that NMES can reduce [153] or prevent [154] muscle wasting in ICU and a recent study demonstrated a reduction in weaning time 
in patients treated with lower limb NMES [154]. The molecular effects of NMES are beginning to be explored. Notably, one study has demonstrated that the application of NMES for $30 \mathrm{~min} \cdot \mathrm{day}^{-1}$ to one leg of a critically ill patient reduced ubiquitin activation and increased total mRNA, IGF-1 and protein content in muscle biopsies compared with a shamtreated control [153]. There is more work to do in this area, both to identify potentially exploitable pathways and to develop appropriate physical interventions.

\section{CONCLUSION}

Underlying the pathogenesis of ICUAW are complex interactions between several different aetiological factors and many levels of muscle function. What is seen at the bedside and the conditions created in the lab do not always correlate. This is reflected in the fact that many of the potentially pathological molecular mechanisms that have been demonstrated in experimental models have yet to be proven to be of relevance in ICUAW.

Clearly, avoidance and early treatment of sepsis and inflammation are highly desirable and may reduce the severity or incidence of ICUAW. Increasing physical activity, potentially with NMES but currently with physiotherapy, may also be beneficial. Tight glycaemic control also prevents ICUAW development, but it has potential side-effects and requires careful use. These strategies are limited and due to the nature of this disease there is no single obvious disease-specific therapeutic target or pathway. Inhibitors of many of the pathological mediators, such as calpain [120] and caspase [70], do exist, raising the possibility that they could contribute to treatment of the disease. However, these targets, like nitric oxide synthase and IGF-1, are ubquitous and therefore their manipulation may have wide-reaching, as-yet unappreciated effects. Myostatin is thought to be muscle specific and myostatin inhibition, for example in the form of anti-myostatin antibodies, offers a potential therapy [155]. There is also a growing body of evidence that lack of nutritional elements such as glutamine may have a role. Glutamine levels are reduced in critical illness and it also has well-recognised antioxidant properties. However, as yet, there is no direct evidence for it or other antioxidants as a treatment [156]. Better muscle-specific therapeutic targets are needed and in order to identify these, a greater understanding of the pathological mechanisms for the development of ICUAW in humans is urgently required.

\section{SUPPORT STATEMENT}

S. Bloch is funded by the UK Medical Research Council. The project was supported by the National Institute for Health Research Respiratory Disease Biomedical Research Unit at the Royal Brompton and Harefield NHS Foundation Trust and Imperial College (London, UK).

\section{STATEMENT OF INTEREST}

A statement of interest for M.I. Polkey can be found at www.erj. ersjournals.com/site/misc/statements.xhtml

\section{REFERENCES}

1 Fletcher S, Kennedy D, Ghosh I, et al. Persistent neuromuscular and neurophysiologic abnormalities in long-term survivors of prolonged critical illness. Crit Care Med 2003; 31: 1012-1016.
2 De Jonghe B, Sharshar T, Lefaucheur J, et al. Paresis acquired in the intensive care unit: a prospective multicenter study. JAMA 2002; 288: 2859-2867.

3 De Jonghe B, Bastuji-Garin S, Sharshar T, et al. Does ICUacquired paresis lengthen weaning from mechanical ventilation? Intensive Care Med 2004; 30: 1117-1121.

4 Ali N, O'Brien JJ, Hoffmann S, et al. Acquired weakness, handgrip strength, and mortality in critically ill patients. Am J Respir Crit Care Med 2008; 178: 261-268.

5 Tennilä A, Salmi T, Pettilä V, et al. Early signs of critical illness polyneuropathy in ICU patients with systemic inflammatory response syndrome or sepsis. Intensive Care Med 2000; 26: 1360-1363.

6 Surviving Sepsis Campaign. About Sepsis. www.survivingsepsis.org/Introduction/Pages/default.aspx Date last accessed: August 2011.

7 Hospital Episode Statistics. Adult Critical Care Data. http:// www.hesonline.nhs.uk/Ease/servlet/ContentServer?siteID= 1937\&categoryID=1298 Date last accessed: August 2011.

8 Subbe C, Criner G, Baudouin S. Weaning units: lessons from North America? Anaesthesia 2007; 62: 374-380.

9 Witt N, Zochodne D, Bolton C, et al. Peripheral nerve function in sepsis and multiple organ failure. Chest 1991; 99: 176-184.

10 Stevens R, Dowdy D, Michaels R, et al. Neuromuscular dysfunction acquired in critical illness: a systematic review. Intensive Care Med 2007; 33: 1876-1891.

11 Eikermann M, Koch G, Gerwig M, et al. Muscle force and fatigue in patients with sepsis and multiorgan failure. Intensive Care Med 2006; 32: 251-259.

12 Ochala J, Ahlbeck K, Radell PJ, et al. Factors underlying the early limb muscle weakness in acute quadriplegic myopathy using an experimental ICU porcine model. PLoS One 2011; 6: e20876.

13 Lacomis D, Zochodne D, Bird S. Critical illness myopathy. Muscle Nerve 2000; 23: 1785-1788.

14 Sander H, Golden M, Danon M. Quadriplegic areflexic ICU illness: selective thick filament loss and normal nerve histology. Muscle Nerve 2002; 26: 499-505.

15 Larsson L, Li X, Edstrom L, et al. Acute quadriplegia and loss of muscle myosin in patients treated with nondepolarizing neuromuscular blocking agents and corticosteroids: mechanisms at the cellular and molecular levels. Crit Care Med 2000; 28: 34-45.

16 Sher JH, Shafiq SA, Schutta HS. Acute myopathy with selective lysis of myosin-filaments. Neurology 1979; 29: 100-106.

17 Khan J, Harrison T, Rich M. Mechanisms of neuromuscular dysfunction in critical illness. Crit Care Clin 2008; 24: 165-177.

18 Friedrich O. Critical illness myopathy: sepsis-mediated failure of the peripheral nervous system. Eur J Anaesthesiol Suppl 2008; 42: 73-82.

19 Giger JM, Haddad F, Qin AX, et al. Effect of unloading on type I myosin heavy chain gene regulation in rat soleus muscle. J Appl Physiol 2005; 98: 1185-1194.

20 Diaz NL, Finol HJ, Torres SH, et al. Histochemical and ultrastructural study of skeletal muscle in patients with sepsis and multiple organ failure syndrome (MOFS). Histol Histopathol 1998; 13: 121-128.

21 Goldberg AL. Protein turnover in skeletal muscle. II. Effects of denervation and cortisone on protein catabolism in skeletal muscle. J Biol Chem 1969; 244: 3223-3229.

22 Lecker SH. Ubiquitin-protein ligases in muscle wasting: multiple parallel pathways? Curr Opin Clin Nutr Metab Care 2003; 6: 271-275.

23 Gomes MD, Lecker SH, Jagoe RT, et al. Atrogin-1, a musclespecific F-box protein highly expressed during muscle atrophy. Proc Natl Acad Sci USA 2001; 98: 14440-14445.

24 Bodine SC, Latres E, Baumhueter S, et al. Identification of ubiquitin ligases required for skeletal muscle atrophy. Science 2001; 294: 1704-1708. 
25 Ochala J, Gustafson AM, Llano Diez M, et al. Preferential skeletal muscle myosin loss in response to mechanical silencing in a novel rat intensive care unit model: underlying mechanisms. J Physiol 2011; 589: 2007-2026.

26 Ikemoto M, Nikawa T, Takeda S, et al. Space shuttle flight (STS90) enhances degradation of rat myosin heavy chain in association with activation of ubiquitin-proteasome pathway. FASEB J 2001; 15: 1279-1281.

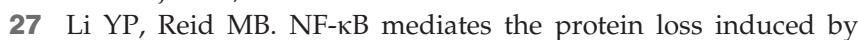
TNF- $\alpha$ in differentiated skeletal muscle myotubes. Am J Physiol Regul Integr Comp Physiol 2000; 279: R1165-R1170.

28 Zhang $\mathrm{P}$, Chen $X$, Fan M. Signaling mechanisms involved in disuse muscle atrophy. Med Hypotheses 2007; 69: 310-321.

29 Cai D, Frantz JD, Tawa NE, et al. IKK $\beta / N F-\kappa B$ activation causes severe muscle wasting in mice. Cell 2004; 119: 285-298.

30 Hunter RB, Stevenson E, Koncarevic A, et al. Activation of an alternative NF- $\kappa B$ pathway in skeletal muscle during disuse atrophy. FASEB J 2002; 16: 529-538.

31 Wray CJ, Mammen JM, Hershko DD, et al. Sepsis upregulates the gene expression of multiple ubiquitin ligases in skeletal muscle. Int J Biochem Cell Biol 2003; 35: 698-705.

32 Combaret L, Taillandier D, Dardevet D, et al. Glucocorticoids regulate mRNA levels for subunits of the $19 \mathrm{~S}$ regulatory complex of the $26 \mathrm{~S}$ proteasome in fast-twitch skeletal muscles. Biochem I 2004; 378: 239-246.

33 Helliwell TR, Wilkinson A, Griffiths RD, et al. Muscle fibre atrophy in critically ill patients is associated with the loss of myosin filaments and the presence of lysosomal enzymes and ubiquitin. Neuropathol Appl Neurobiol 1998; 24: 507-517.

34 Tiao G, Hobler S, Wang JJ, et al. Sepsis is associated with increased mRNAs of the ubiquitin-proteasome proteolytic pathway in human skeletal muscle. J Clin Invest 1997; 99: 163-168.

35 Klaude M, Fredriksson K, Tjader I, et al. Proteasome proteolytic activity in skeletal muscle is increased in patients with sepsis. Clin Sci (Lond) 2007; 112: 499-506.

36 Thomas M, Langley B, Berry C, et al. Myostatin, a negative regulator of muscle growth, functions by inhibiting myoblast proliferation. J Biol Chem 2000; 275: 40235-40243.

37 McFarlane C, Plummer E, Thomas M, et al. Myostatin induces cachexia by activating the ubiquitin proteolytic system through an NF-кB-independent, FoxO1-dependent mechanism. J Cell Physiol 2006; 209: 501-514.

38 Schuelke M, Wagner KR, Stolz LE, et al. Myostatin mutation associated with gross muscle hypertrophy in a child. $N$ Engl J Med 2004; 350: 2682-2688.

39 Lenk K, Schur R, Linke A, et al. Impact of exercise training on myostatin expression in the myocardium and skeletal muscle in a chronic heart failure model. Eur J Heart Fail 2009; 11: 342-348.

40 Liu CM, Yang Z, Liu CW, et al. Myostatin antisense RNAmediated muscle growth in normal and cancer cachexia mice. Gene Ther 2008; 15: 155-160.

41 Gonzalez-Cadavid NF, Taylor WE, Yarasheski K, et al. Organization of the human myostatin gene and expression in healthy men and HIV-infected men with muscle wasting. Proc Natl Acad Sci USA 1998; 95: 14938-14943.

42 Ma K, Mallidis C, Bhasin S, et al. Glucocorticoid-induced skeletal muscle atrophy is associated with upregulation of myostatin gene expression. Am J Physiol Endocrinol Metab 2003; 285: E363-E371.

43 Man WD-C, Natanek SA, Riddoch-Contreras J, et al. Quadriceps myostatin expression in COPD. Eur Respir J 2010; 36: 686-688.

44 Ruff RL, Secrist D. Inhibitors of prostaglandin synthesis or cathepsin B prevent muscle wasting due to sepsis in the rat. J Clin Invest 1984; 73: 1483-1486.

45 Petersson B, Wernerman J, Waller SO, et al. Elective abdominal surgery depresses muscle protein synthesis and increases subjective fatigue: effects lasting more than 30 days. Br J Surg 1990; 77: 796-800.

46 Essen P, McNurlan MA, Gamrin L, et al. Tissue protein synthesis rates in critically ill patients. Crit Care Med 1998; 26: 92-100.

47 Gamrin L, Essen P, Hultman E, et al. Protein-sparing effect in skeletal muscle of growth hormone treatment in critically ill patients. Ann Surg 2000; 231: 577-586.

48 Lang $\mathrm{CH}$, Frost RA. Role of growth hormone, insulin-like growth factor-I, and insulin-like growth factor binding proteins in the catabolic response to injury and infection. Curr Opin Clin Nutr Metab Care 2002; 5: 271-279.

49 Lang $\mathrm{CH}$, Frost RA, Nairn AC, et al. TNF- $\alpha$ impairs heart and skeletal muscle protein synthesis by altering translation initiation. Am J Physiol Endocrinol Metab 2002; 282: E336-E347.

50 Hornberger TA, Hunter RB, Kandarian SC, et al. Regulation of translation factors during hindlimb unloading and denervation of skeletal muscle in rats. Am J Physiol Cell Physiol 2001; 281: C179-C187.

51 Vesali RF, Cibicek N, Jakobsson T, et al. Protein metabolism in leg muscle following an endotoxin injection in healthy volunteers. Clin Sci (Lond) 2010; 118: 421-427.

52 Otto A, Patel K. Signalling and the control of skeletal muscle size. Exp Cell Res 2010; 316: 3059-3066.

53 Bodine SC, Stitt TN, Gonzalez M, et al. Akt/mTOR pathway is a crucial regulator of skeletal muscle hypertrophy and can prevent muscle atrophy in vivo. Nat Cell Biol 2001; 3: 1014-1019.

54 Glass DJ. Molecular mechanisms modulating muscle mass. Trends Mol Med 2003; 9: 344-350.

55 Sacheck JM, Ohtsuka A, McLary SC, et al. IGF-I stimulates muscle growth by suppressing protein breakdown and expression of atrophy-related ubiquitin ligases, atrogin-1 and MuRF1. Am J Physiol Endocrinol Metab 2004; 287: E591-E601.

56 Stitt TN, Drujan D, Clarke BA, et al. The IGF-1/PI3K/Akt pathway prevents expression of muscle atrophy-induced ubiquitin ligases by inhibiting FOXO transcription factors. Mol Cell 2004; 14: 395-403.

57 Lin MC, Leung SY, Fang WF, et al. Down-regulation of insulinlike growth factor I (IGF-I) in the mouse diaphragm during sepsis. Chang Gung Med J 2010; 33: 501-508.

58 Nystrom G, Pruznak A, Huber D, et al. Local insulin-like growth factor I prevents sepsis-induced muscle atrophy. Metabolism 2009; 58: 787-797.

59 Lang CH, Frost RA. Sepsis-induced suppression of skeletal muscle translation initiation mediated by tumor necrosis factor a. Metabolism 2007; 56: 49-57.

60 Crossland H, Constantin-Teodosiu D, Gardiner SM, et al. A potential role for Akt/FOXO signalling in both protein loss and the impairment of muscle carbohydrate oxidation during sepsis in rodent skeletal muscle. J Physiol 2008; 586: 5589-5600.

61 West DW, Burd NA, Staples AW, et al. Human exercisemediated skeletal muscle hypertrophy is an intrinsic process. Int J Biochem Cell Biol 2010; 42: 1371-1375.

62 Sharshar TS, Bastuji-Garin B, De Jonghe RD, et al. Hormonal status and ICU-acquired paresis in critically ill patients. Intensive Care Med 2010; 36: 1318-1326.

63 Glover EI, Phillips SM, Oates BR, et al. Immobilization induces anabolic resistance in human myofibrillar protein synthesis with low and high dose amino acid infusion. J Physiol 2008; 586 6049-6061.

64 Rennie MJ. Anabolic resistance in critically ill patients. Crit Care Med 2009; 37: Suppl. 10, S398-S399.

65 Clark MG, Rattigan S, Barrett EJ. Nutritive blood flow as an essential element supporting muscle anabolism. Curr Opin Clin Nutr Metab Care 2006; 9: 185-189.

66 Ahlbeck K, Fredriksson K, Rooyackers O, et al. Signs of critical illness polyneuropathy and myopathy can be seen early in the ICU course. Acta Anaesthesiol Scand 2009; 53: 717-723. 
67 Leon A, Boczkowski J, Dureuil B, et al. Effects of endotoxic shock on diaphragmatic function in mechanically ventilated rats. J Appl Physiol 1992; 72: 1466-1472.

68 Lanone S, Taille C, Boczkowski J, et al. Diaphragmatic fatigue during sepsis and septic shock. Intensive Care Med 2005; 31: 1611-1617.

69 Supinski G, Nethery D, Stofan D, et al. Comparison of the effects of endotoxin on limb, respiratory, and cardiac muscles. J Appl Physiol 1996; 81: 1370-1378.

70 Supinski GS, Callahan LA. Caspase activation contributes to endotoxin-induced diaphragm weakness. J Appl Physiol 2006; 100: 1770-1777.

71 Polkey MI, Moxham J. Clinical aspects of respiratory muscle dysfunction in the critically ill. Chest 2001; 119: 926-939.

72 Bolton C, Gilbert J, Hahn A, et al. Polyneuropathy in critically ill patients. J Neurol Neurosurg Psychiatry 1984; 47: 1223-1231.

73 Massa R, Carpenter S, Holland P, et al. Loss and renewal of thick myofilaments in glucocorticoid-treated rat soleus after denervation and reinnervation. Muscle Nerve 1992; 15: 1290-1298.

74 Jaber S, Jung B, Matecki S, et al. Clinical review: ventilatorinduced diaphragmatic dysfunction - human studies confirm animal model findings!. Crit Care 2011; 15: 206.

75 Polkey MI, Duguet A, Luo Y, et al. Anterior magnetic phrenic nerve stimulation: laboratory and clinical evaluation. Intensive Care Med 2000; 26: 1065-1075.

76 Mills GH, Ponte J, Hamnegard $\mathrm{CH}$, et al. Tracheal tube pressure change during magnetic stimulation of the phrenic nerves as an indicator of diaphragm strength on the intensive care unit. $\mathrm{Br}$ I Anaesth 2001; 87: 876-884.

77 Hermans G, Agten A, Testelmans D, et al. Increased duration of mechanical ventilation is associated with decreased diaphragmatic force: a prospective observational study. Crit Care 2011; 14: R127.

78 Jung B, Constantin JM, Rossel N, et al. Adaptive support ventilation prevents ventilator-induced diaphragmatic dysfunction in piglet: an in vivo and in vitro study. Anesthesiology 2011; 112: $1435-1443$

79 Gayan-Ramirez G, Testelmans D, Maes K, et al. Intermittent spontaneous breathing protects the rat diaphragm from mechanical ventilation effects. Crit Care Med 2005; 33: 2804-2809.

80 Reid MB, Lannergren J, Westerblad H. Respiratory and limb muscle weakness induced by tumor necrosis factor- $\alpha$ : involvement of muscle myofilaments. Am J Respir Crit Care Med 2002; 166: 479-484.

81 Smith IJ, Lecker SH, Hasselgren PO. Calpain activity and muscle wasting in sepsis. Am J Physiol Endocrinol Metab 2008; 295: E762-E771.

82 Callahan LA, Supinski GS. Sepsis-induced myopathy. Crit Care Med 2009; 37: Suppl. 10, S354-S367.

83 Bartoli M, Richard I. Calpains in muscle wasting. Int J Biochem Cell Biol 2005; 37: 2115-2133.

84 Stockholm D, Herasse M, Marchand S, et al. Calpain 3 mRNA expression in mice after denervation and during muscle regeneration. Am J Physiol Cell Physiol 2001; 280: C1561-C1569.

85 Williams AB, Decourten-Myers GM, Fischer JE, et al. Sepsis stimulates release of myofilaments in skeletal muscle by a calcium-dependent mechanism. FASEB J 1999; 13: 1435-1443.

86 Benson DW, Hasselgren PO, Hiyama DT, et al. Effect of sepsis on calcium uptake and content in skeletal muscle and regulation in vitro by calcium of total and myofibrillar protein breakdown in control and septic muscle: results from a preliminary study. Surgery 1989; 106: 87-93.

87 Smith IJ, Dodd SL. Calpain activation causes a proteasomedependent increase in protein degradation and inhibits the Akt signalling pathway in rat diaphragm muscle. Exp Physiol 2007; 92: $561-573$
88 Supinski GS, Callahan LA. Calpain activation contributes to endotoxin-induced diaphragmatic dysfunction. Am J Respir Cell Mol Biol 2010; 42: 80-87.

89 Supinski GS, Wang W, Callahan LA. Caspase and calpain activation both contribute to sepsis-induced diaphragmatic weakness. J Appl Physiol 2009; 107: 1389-1396.

90 Liu SH, Lai JL, Yang RS, et al. Nitric oxide is not involved in the endotoxemia-induced alterations in $\mathrm{Ca}^{2+}$ and ryanodine responses in mouse diaphragms. Naunyn Schmiedebergs Arch Pharmacol 2002; 366: 327-334.

91 Zink W, Kaess M, Hofer S, et al. Alterations in intracellular $\mathrm{Ca}^{2+}$ homeostasis of skeletal muscle fibers during sepsis. Crit Care Med 2008; 36: 1559-1563.

92 Friedrich $\mathrm{O}$, Hund E, Weber C, et al. Critical illness myopathy serum fractions affect membrane excitability and intracellular calcium release in mammalian skeletal muscle. J Neurol 2004; 251: 53-65.

93 Z'Graggen W, Lin C, Howard R, et al. Nerve excitability changes in critical illness polyneuropathy. Brain 2006; 129: 2461-2470.

94 Rich MM, Teener JWT, Raps EC, et al. Muscle is electrically inexcitable in acute quadriplegic myopathy. Neurology 1996; 46: 731-736.

95 Lefaucheur JP, Nordine $\mathrm{T}$, Rodriguez $\mathrm{P}$, et al. Origin of ICU acquired paresis determined by direct muscle stimulation. Neurol Neurosurg Psychiatry 2006; 77: 500-506.

96 Latronico N, Fenzi F, Recupero D, et al. Critical illness myopathy and neuropathy. Lancet 1996; 347: 1579-1582.

97 Druschky A, Herkert M, Radespiel-Tröger M, et al. Critical illness polyneuropathy: clinical findings and cell culture assay of neurotoxicity assessed by a prospective study. Intensive Care Med 2001; 27: 686-693.

98 Hermans G, De Jonghe B, Bruyninckx F, et al. Clinical review: critical illness polyneuropathy and myopathy. Crit Care 2008; 12: 238 .

99 Rich MM, McGarvey ML, Teener JW, et al. ECG changes during septic shock. Cardiology 2002; 97: 187-196.

100 Rich M, Pinter M, Kraner S, et al. Loss of electrical excitability in an animal model of acute quadriplegic myopathy. Ann Neurol 1998; 43: 171-179.

101 Lin MC, Ebihara S, El Dwairi Q, et al. Diaphragm sarcolemmal injury is induced by sepsis and alleviated by nitric oxide synthase inhibition. Am J Respir Crit Care Med 1998; 158: 1656-1663.

102 Teener JW, Rich MM, Dysregulation of sodium channel gating in critical illness myopathy. J Muscle Res Cell Motil 2006; 27 291-296.

103 Rossignol B, Gueret G, Pennec JP, et al. Effects of chronic sepsis on the voltage-gated sodium channel in isolated rat muscle fibers. Crit Care Med 2007; 35: 351-357.

104 Haeseler G, Foadi N, Wiegand E, et al. Endotoxin reduces availability of voltage-gated human skeletal muscle sodium channels at depolarized membrane potentials. Crit Care Med 2008; 36: 1239-1247.

105 Friedrich O. Critical illness myopathy: what is happening? Curr Opin Clin Nutr Metab Care 2006; 9: 403-409.

106 Caquelard F, Burnet $\mathrm{H}$, Tagliarini F, et al. Effects of prolonged hypobaric hypoxia on human skeletal muscle function and electromyographic events. Clin Sci (Lond) 2000; 98: 329-337.

107 Hoppeler H, Kleinert E, Schlegel C, et al. Morphological adaptations of human skeletal muscle to chronic hypoxia. Int J Sports Med 1990; 11: Suppl. 1, S3-S9.

108 Gosker HR, van Mameren H, van Dijk PJ, et al. Skeletal muscle fibre-type shifting and metabolic profile in patients with chronic obstructive pulmonary disease. Eur Respir J 2002; 19: 617-625.

109 Vianna LG, Koulouris N, Lanigan C, et al. Effect of acute hypercapnia on limb muscle contractility in humans. J Appl Physiol 1990; 69: 1486-1493. 
110 Juan G, Calverley P, Talamo C, et al. Effect of carbon dioxide on diaphragmatic function in human beings. N Engl J Med 1984; 310: 874-879.

111 Rafferty GF, Lou Harris M, Polkey MI, et al. Effect of hypercapnia on maximal voluntary ventilation and diaphragm fatigue in normal humans. Am J Respir Crit Care Med 1999; 160: 1567-1571.

112 Callahan LA, Stofan DA, Szweda LI, et al. Free radicals alter maximal diaphragmatic mitochondrial oxygen consumption in endotoxin-induced sepsis. Free Radic Biol Med 2001; 30: 129-138.

113 Boczkowski J, Lisdero CL, Lanone S, et al. Endogenous peroxynitrite mediates mitochondrial dysfunction in rat diaphragm during endotoxemia. FASEB J 1999; 13: 1637-1646.

114 Callahan LA, Supinski GS. Sepsis induces diaphragm electron transport chain dysfunction and protein depletion. Am J Respir Crit Care Med 2005; 172: 861-868.

115 Callahan LA, Supinski GS. Downregulation of diaphragm electron transport chain and glycolytic enzyme gene expression in sepsis. J Appl Physiol 2005; 99: 1120-1126.

116 Brealey D, Karyampudi S, Jacques TS, et al. Mitochondrial dysfunction in a long-term rodent model of sepsis and organ failure. Am J Physiol Regul Integr Comp Physiol 2004; 286: R491-R497.

117 Fredriksson K, Tjader I, Keller P, et al. Dysregulation of mitochondrial dynamics and the muscle transcriptome in ICU patients suffering from sepsis induced multiple organ failure. PLoS One 2008; 3: e3686.

118 Brealey D, Brand M, Hargreaves I, et al. Association between mitochondrial dysfunction and severity and outcome of septic shock. Lancet 2002; 360: 219-223.

119 Vanhorebeek I, De Vos R, Mesotten D, et al. Protection of hepatocyte mitochondrial ultrastructure and function by strict blood glucose control with insulin in critically ill patients. Lancet 2005; 365: 53-59.

120 Fareed MU, Evenson AR, Wei W, et al. Treatment of rats with calpain inhibitors prevents sepsis-induced muscle proteolysis independent of atrogin-1/MAFbx and MuRF1 expression. Am J Physiol Regul Integr Comp Physiol 2006; 290: R1589-R1597.

$121 \mathrm{Li}$ YP, Chen Y, Li AS, et al. Hydrogen peroxide stimulates ubiquitin-conjugating activity and expression of genes for specific E2 and E3 proteins in skeletal muscle myotubes. Am J Physiol Cell Physiol 2003; 285: C806-C812.

122 Shang F, Gong X, Taylor A. Activity of ubiquitin-dependent pathway in response to oxidative stress. Ubiquitin-activating enzyme is transiently up-regulated. J Biol Chem 1997; 272: 23086-23093.

123 Pickering AM, Koop AL, Teoh CY, et al. The immunoproteasome, the $20 \mathrm{~S}$ proteasome and the PA28 $\alpha \beta$ proteasome regulator are oxidative-stress-adaptive proteolytic complexes. Biochem J 2010; 432: 585-594.

124 McClung JM, Judge AR, Talbert EE, et al. Calpain-1 is required for hydrogen peroxide-induced myotube atrophy. Am J Physiol Cell Physiol 2009; 296: C363-C371.

125 Jiang B, Xiao W, Shi Y, et al. Role of Smac/DIABLO in hydrogen peroxide-induced apoptosis in C2C12 myogenic cells. Free Radic Biol Med 2005; 39: 658-667.

126 Supinski GS, Callahan LA. Free radical-mediated skeletal muscle dysfunction in inflammatory conditions. J Appl Physiol 2007; 102: 2056-2063.

127 Reid MB, Andrade FH, Balke CW, et al. Redox mechanisms of muscle dysfunction in inflammatory disease. Phys Med Rehabil Clin N Am 2005; 16: 925-949.

128 Javesghani D, Magder SA, Barreiro E, et al. Molecular characterization of a superoxide-generating $\mathrm{NAD}(\mathrm{P}) \mathrm{H}$ oxidase in the ventilatory muscles. Am J Respir Crit Care Med 2002; 165: 412-418.
129 Boveris A, Alvarez S, Navarro A, The role of mitochondrial nitric oxide synthase in inflammation and septic shock. Free Radic Biol Med 2002; 33: 1186-1193.

130 Buck M, Chojkier M. Muscle wasting and dedifferentiation induced by oxidative stress in a murine model of cachexia is prevented by inhibitors of nitric oxide synthesis and antioxidants. EMBO J 1996; 15: 1753-1765.

131 Supinski G, Nethery D, Nosek TM, et al. Endotoxin administration alters the force $v s$. pCa relationship of skeletal muscle fibers. Am J Physiol Regul Integr Comp Physiol 2000; 278: R891-R896.

132 Bonetto A, Penna F, Muscaritoli M, et al. Are antioxidants useful for treating skeletal muscle atrophy? Free Radic Biol Med 2009; 47: 906-916.

133 Van den Berghe G, Schoonheydt K, Becx P, et al. Insulin therapy protects the central and peripheral nervous system of intensive care patients. Neurology 2005; 64: 1348-1353.

134 Hermans G, Wilmer A, Meersseman W, et al. Impact of intensive insulin therapy on neuromuscular complications and ventilator dependency in the medical intensive care unit. Am J Respir Crit Care Med 2007; 175: 480-489.

135 Gore DC, Wolf SE, Sanford AP, et al. Extremity hyperinsulinemia stimulates muscle protein synthesis in severely injured patients. Am J Physiol Endocrinol Metab 2004; 286: E529-E534.

136 Cleveland BM, Weber GM. Effects of insulin-like growth factor-I, insulin, and leucine on protein turnover and ubiquitin ligase expression in rainbow trout primary myocytes. Am J Physiol Regul Integr Comp Physiol 2010; 298: R341-R350.

137 Hansen TK, Thiel S, Wouters PJ, et al. Intensive insulin therapy exerts antiinflammatory effects in critically ill patients and counteracts the adverse effect of low mannose-binding lectin levels. J Clin Endocrinol Metab 2003; 88: 1082-1088.

138 Mesotten D, Swinnen JV, Vanderhoydonc F, et al. Contribution of circulating lipids to the improved outcome of critical illness by glycemic control with intensive insulin therapy. J Clin Endocrinol Metab 2004; 89: 219-226.

139 Ishii DN, Lupien SB. Insulin-like growth factors protect against diabetic neuropathy: effects on sensory nerve regeneration in rats. J Neurosci Res 1995; 40: 138-144.

140 Biolo G, De Cicco M, Lorenzon S, et al. Treating hyperglycemia improves skeletal muscle protein metabolism in cancer patients after major surgery. Crit Care Med 2008; 36: 1768-1775.

141 Jespersen JG, Nedergaard A, Reitelseder S, et al. Activated protein synthesis and suppressed protein breakdown signaling in skeletal muscle of critically ill patients. PLoS One 2011; 6: e18090.

142 De Jonghe B, Sharshar T, Hopkinson N, et al. Paresis following mechanical ventilation. Curr Opin Crit Care 2004; 10: 47-52.

143 Berg H, Larsson L, Tesch P. Lower limb skeletal muscle function after 6 wk of bed rest. J Appl Physiol 1997; 82: 182-188.

144 Fink H, Helming M, Unterbuchner C, et al. Systemic inflammatory response syndrome increases immobility-induced neuromuscular weakness. Crit Care Med 2008; 36: 910-916.

145 Lawler JM, Song W, Demaree SR. Hindlimb unloading increases oxidative stress and disrupts antioxidant capacity in skeletal muscle. Free Radic Biol Med 2003; 35: 9-16.

146 Enns DL, Raastad T, Ugelstad I, et al. Calpain/calpastatin activities and substrate depletion patterns during hindlimb unweighting and reweighting in skeletal muscle. Eur J Appl Physiol 2007; 100: 445-455.

147 Sandri M. Apoptotic signaling in skeletal muscle fibers during atrophy. Curr Opin Clin Nutr Metab Care 2002; 5: 249-253.

148 Siu PM, Pistilli EE, Alway SE. Apoptotic responses to hindlimb suspension in gastrocnemius muscles from young adult and aged rats. Am J Physiol Regul Integr Comp Physiol 2005; 289: R1015-R1026. 
149 Fan E, Zanni J, Dennison C, et al. Critical illness neuromyopathy and muscle weakness in patients in the intensive care unit. AACN Adv Crit Care, 20: 243-253.

150 Murray M, Brull S, Bolton C. Brief review: nondepolarizing neuromuscular blocking drugs and critical illness myopathy. Can J Anaesth 2006; 53: 1148-1156.

151 Fan E. What is stopping us from early mobility in the intensive care unit? Crit Care Med 2010; 38: 2254-2255.

152 Morris PE, Griffin L, Berry M, et al. Receiving early mobility during an intensive care unit admission is a predictor of improved outcomes in acute respiratory failure. Am J Med Sci 2011; 341: 373-377.

153 Strasser EM, Stattner S, Karner J, et al. Neuromuscular electrical stimulation reduces skeletal muscle protein degradation and stimulates insulin-like growth factors in an age- and currentdependent manner: a randomized, controlled clinical trial in major abdominal surgical patients. Ann Surg 2009; 249: 738-743.

154 Routsi C, Gerovasili V, Vasileiadis I, et al. Electrical muscle stimulation prevents critical illness polyneuromyopathy: a randomized parallel intervention trial. Crit Care 2010; 14: R74.

155 Haidet AM, Rizo L, Handy C, et al. Long-term enhancement of skeletal muscle mass and strength by single gene administration of myostatin inhibitors. Proc Natl Acad Sci USA 2008; 105: 4318-4322.

156 Hermans G, De Jonghe B, Bruyninckx F, et al. Interventions for preventing critical illness polyneuropathy and critical illness myopathy. Cochrane Database Syst Rev 2009; 1: CD006832. 